\title{
Comparative study on neutron data in integral experiments of MYRRHA mockup critical cores in the VENUS-F reactor
}

\author{
Antonín Krása $^{1, \text { a }}$, Anatoly Kochetkov ${ }^{1}$, Peter Baeten ${ }^{1}$, Guido Vittiglio ${ }^{1}$, Jan Wagemans ${ }^{1}$, and Vicente Bécares ${ }^{2}$ \\ 1 Advanced Nuclear Systems Institute, Belgian Nuclear Research Centre SCK•CEN, Mol, Belgium \\ 2 Nuclear Fission Division, CIEMAT, Madrid, Spain
}

\begin{abstract}
VENUS-F is a fast, zero-power reactor with $30 \%$ wt. metallic uranium fuel and solid lead as coolant simulator. It serves as a mockup of the MYRRHA reactor core. This paper describes integral experiments performed in two critical VENUS-F core configurations (with and without graphite reflector). Discrepancies between experiments and Monte Carlo calculations (MCNP5) of $k_{\text {eff }}$, fission rate spatial distribution and reactivity effects (lead void and fuel Doppler) depending on a nuclear data library used (JENDL-4.0, ENDF-B-VII.1, JEFF-3.1.2, 3.2, 3.3T2) are presented.
\end{abstract}

\section{Introduction}

VENUS-F [1] fast, zero-power reactor core was assembled within the GUINEVERE FP6 EC program (2008-2011) [2] at the Belgian Nuclear Research Centre SCK•CEN. Within the FREYA FP7 EC project (2011-2016) [3] the reactor was coupled to an accelerator with a neutron producing target, making up an Accelerator Driven System (ADS). The main aims were to validate online reactivity monitoring techniques and nuclear data for MYRRHA [4], which will be a fast ADS demonstrator with the option to be also operated in a critical mode.

Fast neutron integral experiments were performed on five critical VENUS-F configurations to address (part of) the nuclear data needs for the neutronic design of the MYRRHA and ALFRED reactor [5]. The VENUS$F$ critical cores were thoroughly characterized and the following parameters were measured:

- global parameters - criticality $\left(k_{\text {eff }}\right)$, kinetic parameters $\left(\beta_{\text {eff }}, \Lambda_{\text {eff }}\right)$,

- local parameters - worth of control and safety rods, spectral indices, spatial fission rate distributions,

- reactivity effects - coolant void, fuel temperature.

In this paper, several selected parameters measured in two of the critical VENUS-F configurations are compared with MCNP5 calculations using general-purpose nuclear data libraries (JEFF, ENDF-B, JENDL).

A detailed analysis of spectral index measurements performed in all VENUS-F critical cores is presented in another article of these proceedings [6].

\section{MYRRHA mockup cores in VENUS-F}

The MYRRHA reactor will use $30 \mathrm{wt} \%$. MOX fuel, lead-bismuth eutectic (LBE) coolant, $\mathrm{BeO}$ reflector and a number of in-pile sections (IPS), see Fig. 1. Because the MOX fuel designed for MYRRHA was not available

a e-mail: akrasa@sckcen.be during the FREYA project, the VENUS-F core has been loaded with $30 \%$ wt. metallic uranium (available from CEA). The uranium fuel together with solid lead (as coolant simulator) and $\mathrm{Al}_{2} \mathrm{O}_{3}$ (used to introduce oxygen that will be present in MYRRHA MOX fuel) make up a VENUS-F fuel assembly (FA), see Fig. 2.

The neutron spectrum in the middle of the VENUSF core is sufficiently similar to the MYRRHA one to serve for nuclear data validation, see Fig. 3. The main differences are situated below $20 \mathrm{keV}$ and around $1 \mathrm{MeV}$ (region of broad resonances in the neutron elastic cross-section on ${ }^{16} \mathrm{O}$ ), where the spectra indicate more neutron moderation in the MYRRHA core.

The VENUS-F reactor consists of a $12 \times 12$ lattice of assemblies surrounded with an outer lead reflector. The lattice is filled with fuel assemblies, lead assemblies (inner reflector), and control and safety rods, see Fig. 4. Two core configurations called $\mathrm{CC} 5$ and $\mathrm{CC} 7$ are described here.

The CC5 VENUS-F core represents a simple core without any perturbation in the active zone, see Fig. 5. It serves as a reference core to be compared with more complicated cores that include various perturbations (reflector, IPS).

The CC7 VENUS-F core is a configuration with a mockup of the MYRRHA $\mathrm{BeO}$ reflector. As $\mathrm{BeO}$ was not available during the FREYA project, graphite was used instead, see Fig. 6. This choice was done because of the similarity of the neutron elastic scattering crosssections, (especially below $350 \mathrm{keV}, \sigma^{\text {el }}\left({ }^{12} \mathrm{C}\right.$ ) is in the middle between $\sigma^{\mathrm{el}}\left({ }^{9} \mathrm{Be}\right)$ and $\left.\sigma^{\mathrm{el}}\left({ }^{16} \mathrm{O}\right)\right)$ and the availability of the reflector grade graphite (from the BR1 reactor).

The neutron spectrum in the middle of the core is purely fast. On the periphery of the active zone the neutron spectrum gets a low-energy tail. In graphite, the neutron spectrum becomes partially moderated, see Fig 7 .

\section{Computational tools}

All presented Monte Carlo calculations were performed with the MCNP5-1.60 code [7]. The latest versions and test

(c) The Authors, published by EDP Sciences. This is an Open Access article distributed under the terms of the Creative Commons Attribution License 4.0 (http://creativecommons.org/licenses/by/4.0/). 


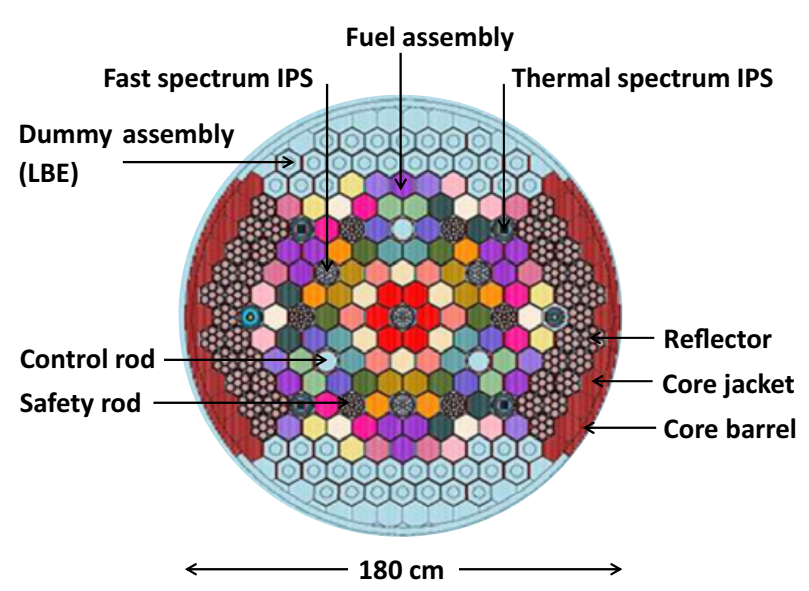

Figure 1. Horizontal cut through an equilibrium critical MYRRHA core. It consists of 108 fuel assemblies (various burnup levels are distinguished by colours), 6 safety rods, 3 control rods, 4 fast spectrum IPS, 6 thermal spectrum IPS, and $\mathrm{BeO}$ reflector assemblies and dummy assemblies filled with LBE (serving partially as a reflector too).

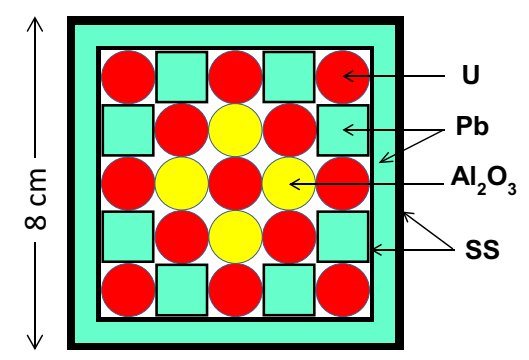

Figure 2. Horizontal cut through a VENUS-F fuel assembly $\left(5 \times 5\right.$ grid of $13 \mathrm{U}+8 \mathrm{~Pb}+4 \mathrm{Al}_{2} \mathrm{O}_{3}$ rodlets surrounded by $\mathrm{Pb}$ plates in a SS casing).

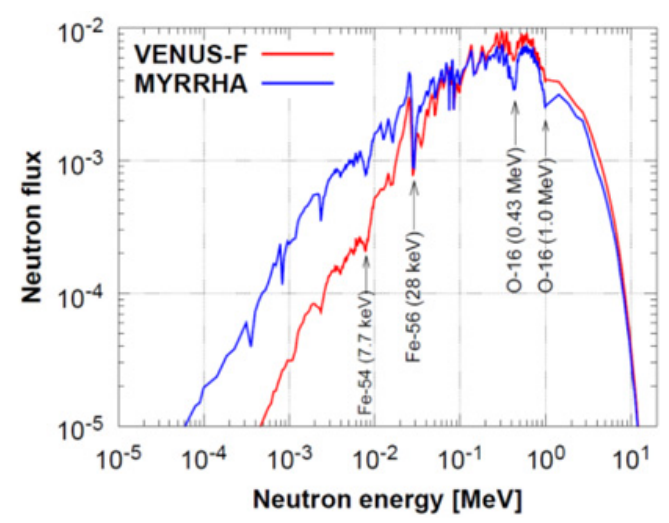

Figure 3. Neutron spectra in the middle of MYRRHA and VENUS-F critical cores. MCNP calculation. The dips corresponding to the resonances in excitation curves of iron and oxygen are indicated.

versions of the three main general-purpose nuclear data libraries were used:

- JENDL-4.0 (released in May 2010) [8],

- ENDF/B-VII.1 (released in Dec 2011) [9],

- JEFF: 3.1.2 (released in Feb 2012), 3.2 (March 2014), the test versions 3.3T1 (March 2016) and 3.3T2 (June 2016) [10]. Calculations with 3.3T1 lead to unrealistic results due to a bug (missing background cross-section for $\mathrm{Pb}$ ), which was corrected in 3.3T2. Therefore, the

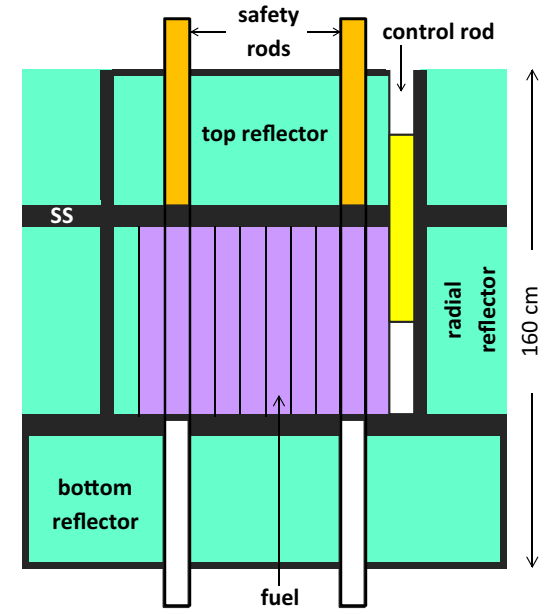

Figure 4. Vertical cut through a critical VENUS-F reactor. View with two safety rods extracted and a control rod partially inserted.

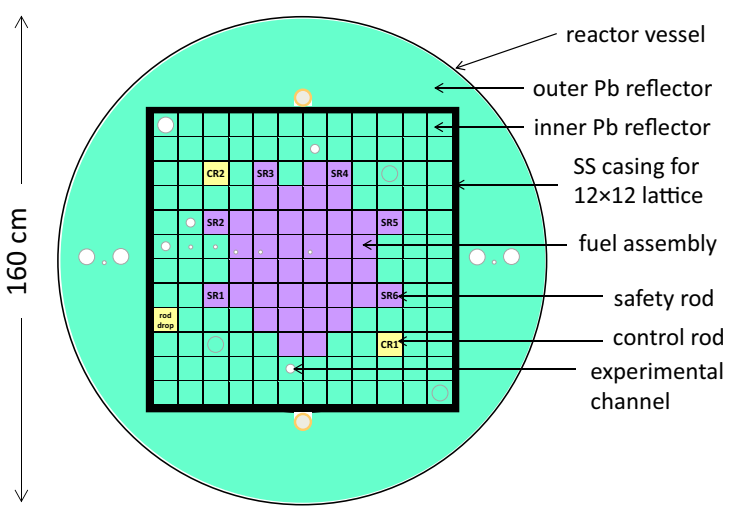

Figure 5. Horizontal cut through the VENUS-F CC5 core. It consists of 144 assemblies: 41 fuel assemblies (including 6 safety rods with fuel followers), 2 control rods, a rod drop system, and 100 lead assemblies.

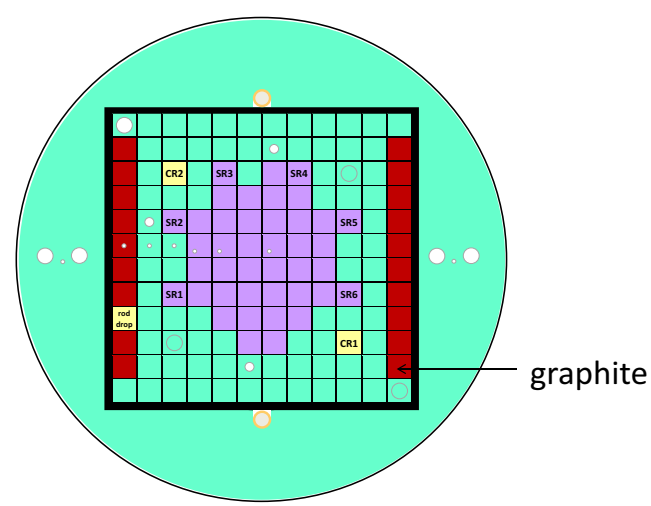

Figure 6. Horizontal cut through the VENUS-F CC7 core. 19 graphite reflector assemblies were added into the CC5 core to simulate the $\mathrm{BeO}$ reflector of MYRRHA (Fig. 1).

results calculated with 3.3T1 are not included in this paper.

Extensive sensitivity and uncertainty analysis has been performed using the SANDY code [11] to identify the uncertainties of which parameters need to be improved in order to reduce the uncertainty in $k_{\text {eff }}$. The results will be published as a separate article. 


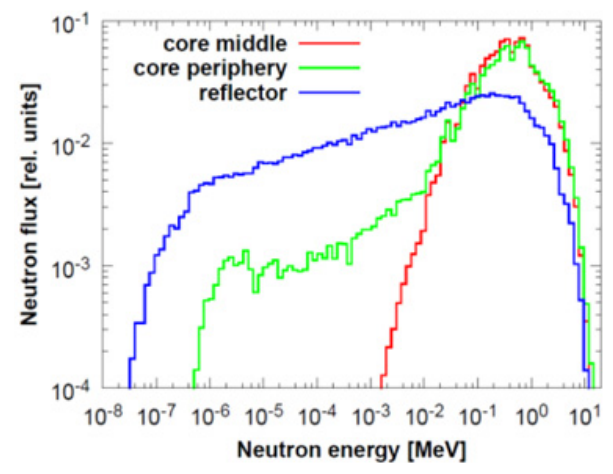

Figure 7. Calculated neutron spectra in the middle of the VENUS-F core, on its periphery and in the graphite reflector.

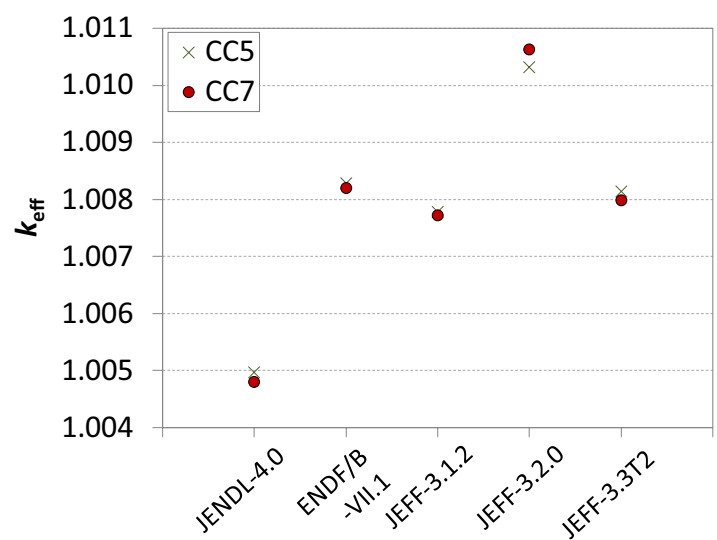

Figure 8. MCNP calculations of $k_{\text {eff }}$ for the $\mathrm{CC} 5$ and $\mathrm{CC} 7$ critical cores. Five nuclear data sets were used.

\section{Results}

Measured $k_{\text {eff }}$, radial fission rate distributions and reactivity effects are compared with calculations in the following subsections.

\subsection{Effective multiplication factor}

For both CC5 and CC7 critical VENUS-F cores, the calculations with any of the nuclear data libraries give $k_{\text {eff }}$ $>1$, see Fig. 8. The overestimation is between 480 and $1060 \mathrm{pcm}$, which is of a similar magnitude as a trend observed in the fast critical MUSE-4 benchmark [12].

For the CC5 core, the best agreement with experiment is obtained with JENDL-4.0 (500 pcm overestimation), the biggest discrepancy is observed with JEFF-3.2 (1030 pcm). JEFF-3.1.2, JEFF-3.3T2 and ENDF/B-VII.1 give around $800 \mathrm{pcm}$ overestimation.

The insertion of graphite (CC7 core) has a minor effect on the overestimation of $k_{\text {eff }}$. For most data sets, it decreases by 5 to $20 \mathrm{pcm}$ (with a precision of $3 \mathrm{pcm}$ ). Only for JEFF-3.2 the effect is opposite: an increase of $k_{\text {eff }}$ by $30 \mathrm{pcm}$.

\subsection{Radial fission rate distribution}

The radial U-235 fission rate distribution through the active zone, and the inner and outer reflector was measured in the CC5 and CC7 cores (see the experimental channels on Fig. 5).

The calculations describe well the shape inside the CC5 active zone but predict a steeper decrease in the lead

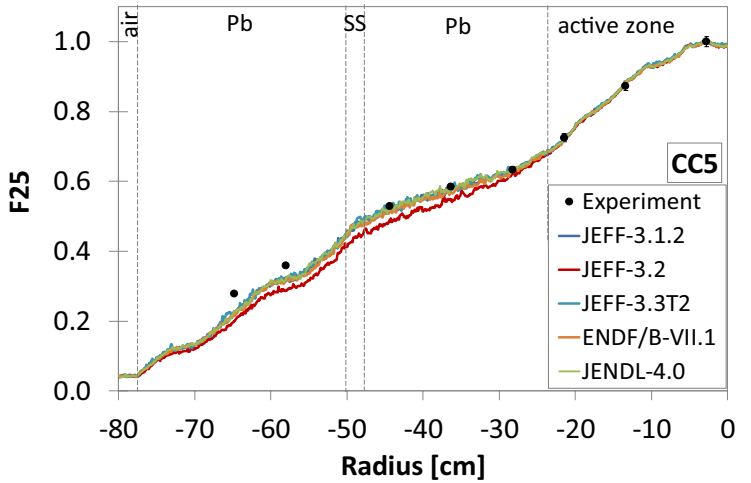

Figure 9. Radial U-235 fission rate distribution normalized to the point closest to the $\mathrm{CC} 5$ core centre. The measurements are compared with MCNP calculations using five nuclear data sets.

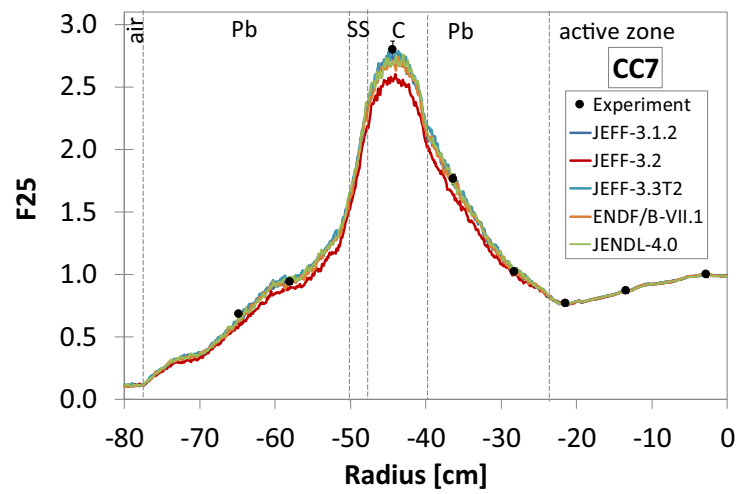

Figure 10. Radial U-235 fission rate distribution normalized to the point closest to the $\mathrm{CC} 7$ core centre. The measurements are compared with MCNP calculations using five nuclear data sets.

reflector, see Fig. 9. The C/E goes down to 0.7 for JEFF3.2 and 0.8 for all other libraries.

The presence of graphite (CC7 core) causes a shift in the neutron spectrum (Fig. 7) and consequently an increase of the U-235 fission rate in the reflector by a factor of 5, see Fig. 10. This behaviour is well modelled with MCNP using any of the nuclear data libraries but JEFF-3.2, which underestimates the influence of graphite by about $10 \%$. Additionally, graphite mitigates the $\mathrm{C} / \mathrm{E}$ discrepancy in the outer $\mathrm{Pb}$ reflector from 0.8 to 0.95 .

\subsection{Lead coolant void reactivity effect}

The lead void effect was measured in the CC7 core using a null-reactivity method (compensation of a reactivity effect by adjustment of accurately calibrated control rods to reach criticality again).

A positive lead void effect (up to $(23 \pm 1)$ pcm) was measured when the middle $20 \mathrm{~cm}$ length $\mathrm{Pb}$ rodlets (Fig. 2) in 1 to 5 central fuel assemblies were replaced with air, see Fig. 11.

The MCNP calculations predict an effect compatible with zero within the statistical uncertainties. Although an MCNP calculation of a reactivity effect (i.e. a difference of two calculations) can hardly give a precision better than $3 \mathrm{pcm}$, it is possible to conclude that MCNP fails to model the lead void effect correctly with any of the applied libraries. In absolute terms, the calculations underestimate the clearly observed positive lead void reactivity effect. 


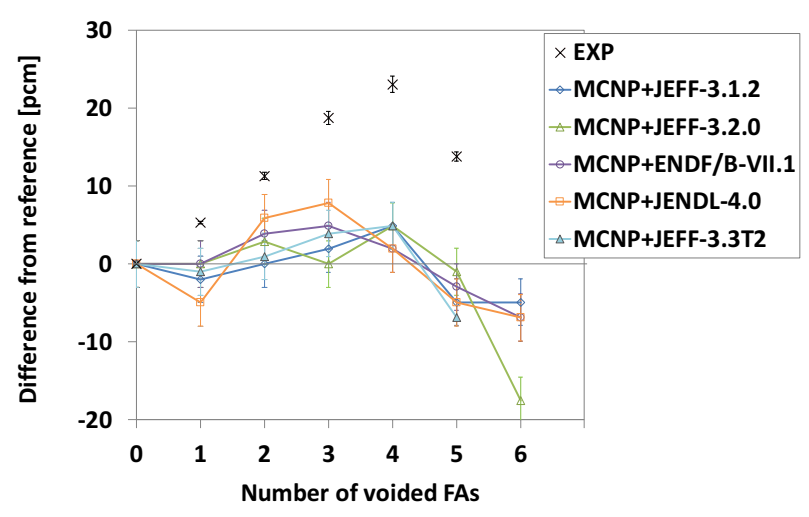

Figure 11. Comparison of the experimental lead coolant void effect in the CC7 core with MCNP calculations using five nuclear data sets. The curves are drawn to guide the reader's eyes.

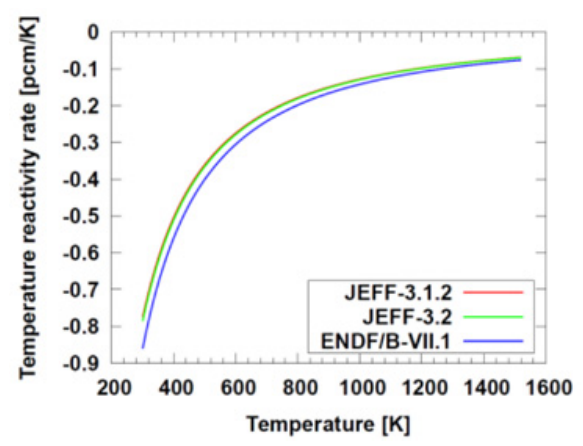

Figure 12. Rate of the temperature reactivity effect. Fit of MCNP calculation using three nuclear data sets.

\subsection{Doppler (fuel temperature) reactivity effect}

A small negative reactivity effect (up to $-(2.9 \pm 0.3) \mathrm{pcm})$ due to a temperature increase by $4 \mathrm{~K}$ was measured in the CC7 core using the null-reactivity method (the same as in Sect. 4.3). Although it is too small to be directly compared with calculations (impossible to reach sufficient precision with a stochastic calculation), the observed rate of the effect $(-0.7 \mathrm{pcm} / \mathrm{K}$ around room temperature) can be compared with a fit of the calculated dependence of $k_{\text {eff }}$ on the fuel temperature $T$.

MCNP calculations were performed in the 300-1500 K range with three nuclear data sets. The calculated rates using the JEFF-3.1.2 and JEFF-3.2 libraries agree with the measured ones at room temperature and quickly decrease with increasing temperature down to $0.07 \mathrm{pcm} / \mathrm{K}$ at $1500 \mathrm{~K}$ (operational fuel temperature for MYRRHA), see Fig. 12. A calculation using ENDF/B-VII.1 predicts a slightly steeper trend around room temperature (about $-0.9 \mathrm{pcm} / \mathrm{K})$.

\section{Conclusion and outlook}

Five nuclear data sets were used in MCNP calculations for comparison with fast neutron integral experiments on two critical VENUS-F cores. Calculations with any of the nuclear data libraries overestimate $k_{\text {eff }}$ (up to about $1000 \mathrm{pcm}$ ). The best result is obtained with JENDL-4.0 (overestimation by about $500 \mathrm{pcm}$ ).

The radial distribution of the U-235 fission rate is well predicted except for the outer $\mathrm{Pb}$ reflector region, where calculations underestimate the experiment. The increase of the fission rate caused by the graphite reflector is well modelled except using JEFF-3.2, which underestimates the effect.

MCNP underestimates the lead void effect with any nuclear data set. The rate of the fuel temperature effect calculated with MCNP is in agreement with the measured one around room temperature.

Contribution of individual isotopes to the calculated quantities is to be investigated. Further nuclear data studies have been performed on a larger critical VENUS-F core with thermal spectrum islands, which will be published separately.

During the FREYA project, only solid lead was used as a coolant simulator. Because MYRRHA will use LBE coolant, all lead (rods and plates) in the VENUSF fuel assemblies have been replaced with bismuth. An experimental campaign aiming at thorough $\mathrm{Bi}$ data validation was carried out in fall 2016.

This work was supported by the FP7 of the EC through FREYA No. 269665. The first author is grateful to Gašper Žerovnik for useful comments.

\section{References}

[1] A. Kochetkov et al, EPJ Web of Conferences 106, 06004 (2016)

[2] P. Baeten et al., Proceedings of PHYSOR-2008, Interlaken, Switzerland (September 14-19, 2008)

[3] A. Kochetkov et al., Proceeding of TCADS-2, Nantes, France (21-23 May 2013)

[4] G. Van den Eynde et al, J. Nucl. Sci. Technol. (2015) http://dx.doi.org/10.1080/00223131.2015. 1026860

[5] G. Grasso et al., Nucl. Eng. and Design 278, 287-301 (2014)

[6] A. Kochetkov et al., Proceeding of ND2016, Bruges, Belgium (11-16 Sep. 2016)

[7] X-5 Monte Carlo Team, LA-UR-03-1987 (2003)

[8] K. Shibata et al, J. Nucl. Sci. Technol. 48(1), 1-30 (2011)

[9] M.B. Chadwick, Nuclear Data Sheets 112, 2887 2996 (2011)

[10] A. Plompen, Proceeding of ND2016, Bruges, Belgium (11-16 Sep. 2016)

[11] L. Fiorito, JEF/DOC-1726 (2015)

[12] NEA/NSC/DOC(2005)23 